\title{
Raman Spectroscopy-A Powerful Tool for in situ Planetary Science
}

\author{
N. Tarcea • T. Frosch $\cdot$ P. Rösch $\cdot$ M. Hilchenbach • \\ T. Stuffler $\cdot$ S. Hofer $\cdot$ H. Thiele $\cdot$ R. Hochleitner • \\ J. Popp
}

Received: 22 August 2006 / Accepted: 13 September 2007 / Published online: 27 October 2007

(C) Springer Science+Business Media B.V. 2007

\begin{abstract}
This paper introduces Raman spectroscopy and discusses various scenarios where it might be applied to in situ planetary missions. We demonstrate the extensive capabilities of Raman spectroscopy for planetary investigations and argue that this technique is essential for future planetary missions.
\end{abstract}

Keywords Raman spectroscopy $\cdot$ Remote Raman spectroscopy $\cdot$ Space-borne Raman spectrometers $\cdot$ In situ planetary science

\section{Introduction}

In the last few years, Raman spectroscopy (Popp and Kiefer 2000) has been recognized as a possible method for in situ planetary analysis (Cochran 1981; McMillan et al. 1996; Sharma et al. 2002; Tarcea et al. 2002; Ellery and Wynn-Williams 2003; Estec et al. 1972; Isreal et al. 1997; Haskin et al. 1997; Korotev et al. 1998; Edwards et al. 1999; Popp et al. 2001; Wang et al. 1999). Two important fields where Raman spectroscopy is used are mineralogical and organic/biological analysis. It has been shown that Raman spectroscopy-and in particular micro-Raman spectroscopy_can contribute to resolving various questions in

N. Tarcea · T. Frosch · P. Rösch · J. Popp ( $\varangle)$

Institute for Physical Chemistry, University of Jena, Jena, Germany

e-mail: juergen.popp@uni-jena.de

M. Hilchenbach

Max-Plank-Institut für Sonnensystemforschung, Katlenburg-Lindau, Germany

T. Stuffler $\cdot$ S. Hofer $\cdot$ H. Thiele

Kayser-Threde $\mathrm{GmbH}$, Munich, Germany

R. Hochleitner

Mineralogische Staatssammlung, Munich, Germany

J. Popp

Institut für Physikalische Hochtechnologie (IPHT), Jena, Germany 
the field of planetary and asteroid investigations because it allows one to address numerous issues, e.g.:

1. Analysis of mineralogical and geochemical materials from a planetary surface in order to understand a planet's evolutionary history.

2. Identification of inorganic, organic or biological compounds, which facilitates the search for past or present life on remote celestial bodies (e.g., Mars). This is the main benefit offered by Raman spectroscopy.

3. Identification of the principal mineral phases (i.e., those making up at least $90 \%$ of the material in soils and rocks).

4. Classification of rocks (igneous, sedimentary and metamorphic) and definition of petrogenetic processes.

5. Determination of the oxidation state of planetary elements, e.g., soil, rock surfaces and inside rocks, and the ability to finely differentiate among mineral species.

6. Analysis of the content of volatiles and gaseous inclusions $\left(\mathrm{H}_{2} \mathrm{O}, \mathrm{SO}_{3}, \mathrm{CO}_{2}, \mathrm{NO}_{2}\right.$, $\mathrm{H}_{2}, \mathrm{O}_{2}$ ) in minerals and glasses.

7. Determination of selected minor and trace element contents (e.g., rare earth elements).

8. Determination of reaction kinetics, i.e., oxidation processes on newly exposed surfaces and determination of the reaction products.

9. Morphology of organic inclusions (fossils) and minerals on a micrometer scale obtainable by Raman mapping measurements (Tarcea et al. 2003).

10. Identification of water and ice on, e.g., Mars; identification of secondary minerals, clays, state of carbonaceous matter and hydrated crystals.

Furthermore, Raman spectroscopy in general requires only minimal or no sample preparation. Solid, liquid and gaseous samples can be measured as well as transparent or nontransparent samples. Additionally, samples with different surface textures can be analysed. In short, Raman spectroscopy can be applied to any optically accessible sample (i.e., the sample can be reached by the excitation laser beam and the inelastically scattered photons can be collected). Raman spectroscopy also offers measuring configurations that can accommodate target sizes from $1 \mu^{2}$ (standard laboratory Raman microspectroscopy) up to a few $\mathrm{dm}^{2}$, at ranges from a few mm up to $1 \mathrm{~km}$ (Sharma et al. 2003).

Employing Raman spectroscopy for in situ space exploration requires a reliable, automated, sufficiently robust, suitably miniaturized and low-power-consuming instrument capable of addressing the issues enumerated here.

Technical developments have made possible the design of a new generation of small Raman systems which are suitable for robotic deployment on planetary surfaces. Space-borne Raman spectrometers that fulfill these characteristics have been studied, e.g. in the USA for future Mars missions, by Wang and co-workers (1998, 2003). A tiny diode laser (readily available for excitation wavelengths in infrared and visible) serves as the radiation source for this miniaturized Raman spectrometer. For signal detection, a conventional setup was used including spectrometer and CCD. Also, in Germany, a DLR-funded breadboard study called Mineral Investigation by in situ Raman Spectroscopy (MIRAS) was successfully performed under the leadership of the university of Würzburg and Jena in cooperation with industry (Kayser-Threde GmbH) (Popp et al. 2002). For this setup, a diode laser in a Littrow configuration operating at $785 \mathrm{~nm}$ was used. An optical tuneable filter (AOTF) was used as the wavelength selecting and deflecting element, and an avalanche photodiode (APD) point detector was used to detect the scattered light.

A prototype of a miniature laser-Raman spectrometer with an $852 \mathrm{~nm}$ laser, CCD detector system and confocal microscope was developed by Dickensheets et al. (2000). A remote pulsed laser Raman spectroscopy system for mineral analysis on planetary surfaces 
was presented by Sharma et al. (2002). UV resonance Raman has been used for easy identification of endolithic organisms and their background mineral matrix; Storrie-Lombardi et al. (1999) discussed this technique for possible use in a future remote planetary mission. A Raman spectrometer was considered as a candidate instrument (Maurice et al. 2004; AURORA/EXOMARS 2005; Popp et al. 2003; Mugnuolo et al. 2000) for the PASTEUR exobiology multiuser facility and planned for the EXOMARS, the first AURORA mission. For the planned 2013 flight to Mars, a combination of Raman and Laser Induced Breakdown Spectrometer (LIBS) in a complex single instrument is planned (Rull and Martinez-Frias 2006).

The progress in the development of appropriate Raman spectroscopic equipment for extraterrestrial research and the advantages of Raman spectroscopy support the idea that this technique is suited for future planetary missions, where the ability to gather information about the mineralogy and the possible presence of organic species will be critical.

\section{Raman Scattering}

Raman spectroscopy is based on the inelastic scattering of laser light by molecules or crystals. When light interacts with matter, most of the incident light is scattered elastically (Rayleigh scattering) with no change in energy. Only a small amount, $10^{-8}$ to $10^{-12}$, of the incident radiation is modulated by the molecular scattering system. Depending on the coupling, the incident photons either gain or lose energy. A sample model of scattering system is shown in Fig. 1. A photon with the energy $h v_{L}$ is incident on the scattering system with the energy level $h v_{R}=E_{f}-E_{i}$, where $i$ and $f$ label two quantum states. The StokesRaman effect results from the transition from the lower energy level $E_{i}$ to a higher one $\left(E_{f}\right)$.

The anti-Stokes effect transfers energy from the system to the incident light wave, which corresponds to the transition from a higher energy level $\left(E_{f}\right)$ to a lower one $\left(E_{i}\right)$. Since the anti-Stokes scattering occurs from a thermally excited state $\left(E_{f}\right)$ which is, according to Boltzmann statistics, less populated than the ground state $\left(E_{i}\right)$, the anti-Stokes is less than the Stokes intensity. In Fig. 2 a typical Stokes and anti-Stokes Raman spectrum from anatase $\left(\mathrm{TiO}_{2}\right)$ is shown.

Usually the spectral assignment from a Raman spectrum is straightforward. Due to the fingerprint-like information of a vibrational spectrum, and the intrinsic narrow line width of a Raman band, each substance has an easy-to-recognize Raman spectrum. Figure 3 shows
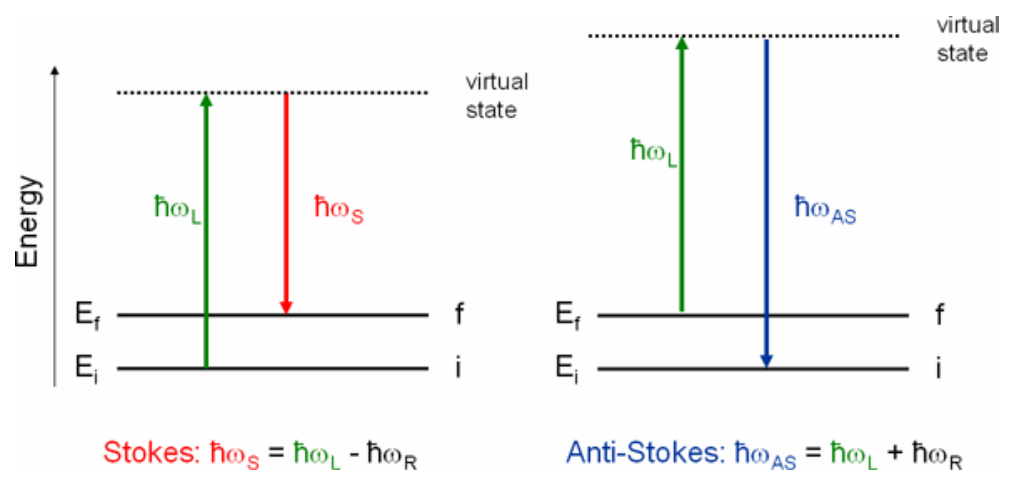

Fig. 1 Raman scattering process 
Fig. 2 Stokes and anti-Stokes Raman spectra of anatase $\left(\mathrm{TiO}_{2}\right)$

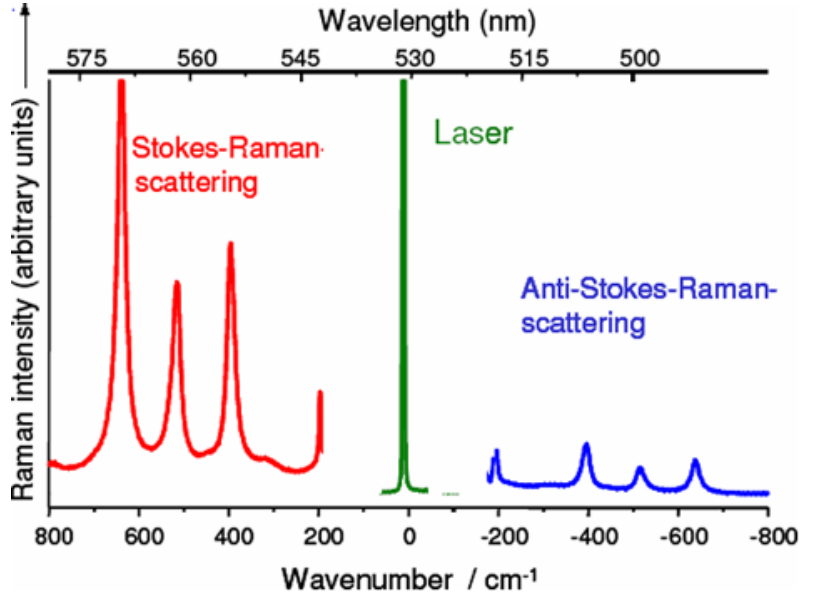

the spectra of some major minerals. Distinctive spectra allow for a quick assignment. Even for the minerals from the same class, e.g., $\mathrm{K}$ feldspar and $\mathrm{Na}$ feldspar, there are important distinct spectral features (e.g. in the region from 300 to $500 \mathrm{~cm}^{-1}$ ).

A schematic diagram of a conventional Raman setup is shown in Fig. 4. A monochromatic laser beam is used for excitation, and the resulting wavelength shifts of the scattered radiation are detected using a dispersion element and a photon detector.

\section{Instrument}

Information about a planetary surface can be gained through orbital and landed Raman spectrometers. Remote Raman spectroscopy from orbiting instruments would be a new tool for the investigation of planets. Klein et al. (2004) presented the results of various feasibility studies commissioned by the European Space Agency to apply a remote Raman measurement device 10-100 m away from the landing site on a planetary body or even from the orbiting instruments. Their studies revealed that remote Raman spectroscopy will be a demanding task. To achieve this goal, the commonly applied laboratory Raman spectroscopy and the well-known Lidar technology need to be combined. Remote-Raman techniques have been evaluated for their potential applications on Mars (Sharma et al. 2002; Lucey et al. 1998; Sharma et al. 2003; Stoper et al. 2004; Misra et al. 2005). While the application of such a planetary remote Raman device relies heavily on future technical developments, the employment of a miniaturized Raman sensor head embedded directly on a lander or a Rover is well established (Cochran 1981; McMillan et al. 1996; Sharma et al. 2002; Tarcea et al. 2002; Ellery and Wynn-Williams 2003; Estec et al. 1972; Isreal et al. 1997; Haskin et al. 1997; Korotev et al. 1998; Edwards et al. 1999; Popp et al. 2001; Wang et al. 1999). For the majority of the proposed Raman devices, the basic design is a modular construction approach. The main components of the final instrument are the laser unit, the Raman head, the Rayleigh filtering box and the spectral sensor (spectrometer with a matching detector). The modularity offers the possibility of basic components being shared among different instruments. There is no fixed configuration for the use of such a spectrometer on a planetary mission. Different configurations are directly linked to the different scenarios of using such a Raman device: 

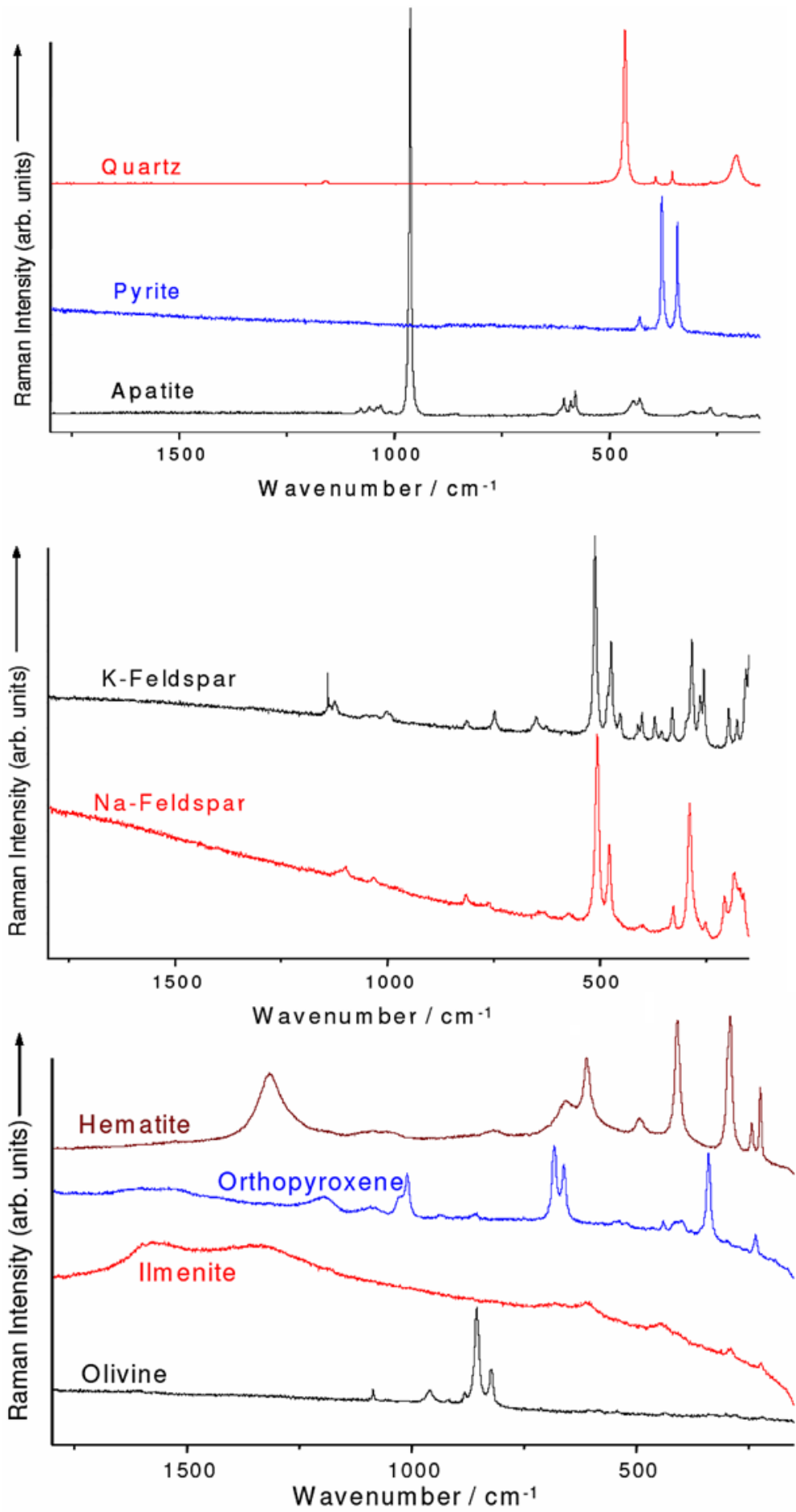

Fig. 3 Raman spectra of different minerals 


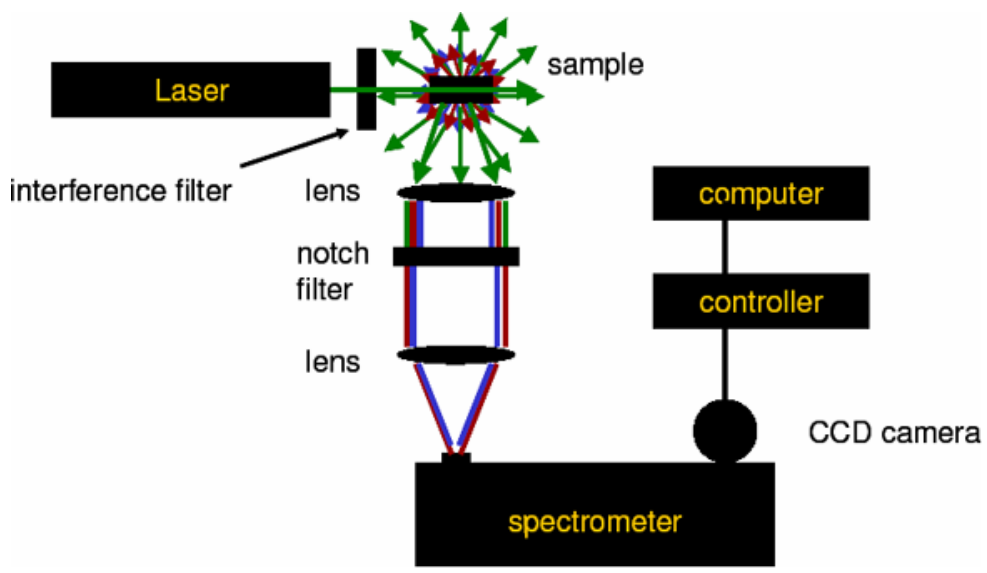

Fig. 4 Principal schematics of an experimental Raman setup

- The whole instrument can be mounted on the planetary lander having a common system for sample retrieval and sample handling. Samples can be shared with other instruments.

- The sensor head is integrated on a lander robotic arm with no electronics or movable parts in the sensor head. The main box is mounted on the lander platform, including light source and spectrometer. The sensor head and the light source, as well as spectrometer are linked via optical fibre.

- The sensor head is integrated into a MOLE (Mobile Penetrometer) (Richter et al. 2002). No electronics or movable parts are placed into the sensor head, the main box being mounted on the lander platform. Optical fibre make the connections with the sensor head.

- The whole device is integrated on the rover (with an optional robotic arm). No electronics or movable parts would be placed in the sensor head. Depending on the needs of the mission, the Raman device can be adapted easily.

Ellery et al. (2004) argued that, for future Mars lander missions, a specially designed Raman spectrometer will be indispensable. The Raman spectrometer will aid studies of Martian mineralogy and astrobiology and outline astrobiologically relevant features of the Martian environment. Of course, it will also meet the requirements for the detection of biotic residues. Specifically, Ellery et al. (2004) introduced a Raman spectrometer combined with a confocal imager instrument that is extremely compact and low mass; thus, it is ideally suited for onsite planetary applications, in particular astrobiological and mineralogical investigations. In their setup, the main electronics - which may be housed in a lander-are separated from the sensor head, which in turn may be incorporated into a probe such as a "ground-penetrating mole" connected by an optical-fibre-based tether. This strategy allows us to examine environments that would otherwise be inaccessible. The authors suggested that in order to detect biomolecules on the surface of Mars, subsurface penetration using such a mole will be essential; a Raman instrument is ideal for such deployment.

The performance of a flight-ready instrument is not expected to match the performance of a standard laboratory instrument, mainly due to constraints imposed by a very limited mission budget for mass/volume and energy (Popp et al. 2001). To close the gap in performance, the problems which are method-inherent (fluorescence, generally low Raman scattering efficiency, etc.), and those problems which are dealt with by technical artifices in the laboratory 
(pulsed excitation and gated detection, extremely sensitive detectors, etc.), have to be minimized for a flight-ready instrument without applying costly (in mass/energy terms) technical tricks.

\subsection{Laser System and Laser Wavelength}

To obtain Raman spectra of sufficient quality, the laser beam quality and shape as well as the selected wavelength play a major role. A continuous wave laser illumination during the measurement is preferred to reduce the necessary laser power and also to keep the overall measurement time as short as possible. Diode lasers are efficient (50\% conversion rates are normal), compact and inexpensive light sources. They are mass-produced for telecom applications, bar code scanners, CD players or computer optical drives. However, their use for Raman spectroscopy has been limited due to the large spectral laser linewidth and the problematic control of the output frequency. The newly developed laser diodes, e.g., ExternalCavity-Diode Lasers (ECDL), Distributed Feed-Back (DFB), Distributed Bragg Reflector (DBR) or even the Laser Diode with External Fibre Bragg Grating partially solve all these problems. Nevertheless. the working characteristics of such a laser vary a great deal with the temperature. Accounting for these laser changes implies the use of an automatic calibration procedure (e.g., a diamond calibration sample) together with very efficient temperature stabilisation for the laser system.

The diode lasers are the best technical option if exciting wavelengths in VIS-NIR are required. Until recently, no compact deep UV diode laser was available on the market which was capable of running at room temperature with an efficiency of more than $15 \%$. A promising solution are the so-called hollow cathode $\mathrm{NeCu}$ ion-lasers, which emit @ $248.6 \mathrm{~nm}$; these were recently released by Photon Systems. The laser was developed partially with regard to compact science instruments for NASA technology programs (Storrie-Lombardi et al. 2001).

Raman scattering is only one of multiple physical processes which might take place when light interacts with matter. Some of these processes are competing with the Raman process (e.g., absorption) and/or are interfering with the detection of the weak Raman photons (e.g., fluorescence). For a given sample, the result of the interaction photon-matter is highly dependent on the wavelength of the photons used in interaction. Therefore, the Raman signal yield and the gained information can be maximized by carefully choosing the excitation laser wavelength in a normal Raman experiment.

For a classic Raman measurement, the only way to get rid of one of the main obstaclesflorescence excitation - is to tune the laser wavelength to a spectral region where the probability of interference from the florescence signal is minimal. Two approaches are normally used. The first and most widely used is to lower the energy of the incoming photon such that the excitation of the molecule in an electronic state does not take place. Therefore, the wavelength of the laser used for excitation is in the NIR region of the spectrum (from $785 \mathrm{~nm}$ up to $1,064 \mathrm{~nm}$ ). Using this approach for most of the samples (especially the biological samples) avoids fluorescence excitation. On the other hand, avoiding fluorescence in this way for the minerals proves inefficient, since in minerals there is always a certain amount of rare-earth elements and impurities which do have the excited electronic levels at relatively low energies. However, the incoming photon energy cannot go arbitrarily low, since excitation at $830 \mathrm{~nm}$ will not allow a full-range Raman spectrum $\left(4,000-100 \mathrm{~cm}^{-1}\right)$ to be fully recorded with a standard CCD camera based on silicon technology (the cutoff wavelength is $\sim 1,100 \mathrm{~nm}$ ). 
The second approach used for minimizing the interference of fluorescence with the Raman signal is to shift the excitation wavelength into the deep UV region. At these wavelengths the fluorescence is excited but no fluorescence interference exists when excitation is at wavelengths below about $250 \mathrm{~nm}$. A typical Raman spectral range of 4,000 cm $\mathrm{cm}^{-1}$ occurs at less than $30 \mathrm{~nm}$ above the excitation wavelength at $250 \mathrm{~nm}$. Independent of the excitation wavelength, almost no material fluoresces at wavelengths below about $280 \mathrm{~nm}$. This provides complete spectral separation of Raman and fluorescence emission bands resulting in high signal-to-noise measurements.

In addition to having the Raman and fluorescence signals spectrally well separated, if the Raman excitation occurs within an electronic resonance band of a material, the scattering cross-section can be improved by as much as $10^{8}$. Diamond, nitrites and nitrates, and many other organic and inorganic materials, have strong absorption bands in the deep UV and exhibit resonance enhancement of Raman bands when excited in the deep UV (Chadha et al. 1993).

Comparing the available Raman signal for both cases of NIR and UV excitation one has to observe that the Raman cross-section itself is dependent on the excitation wavelength to the inverse fourth power resulting in higher Raman intensity with shorter wavelength laser excitation. Therefore, an increase of approximately two orders of magnitude in the Raman scattered photons can be obtained by moving from NIR (at $785 \mathrm{~nm}$ ) to the UV spectral region $(248 \mathrm{~nm})$. In addition, the size of the sampling spot for micro-Raman experiments is proportional to the wavelength of the laser beam, and therefore we can achieve a better spatial resolution for Raman mapping experiments when the excitation laser has a shorter wavelength.

However, using the deep UV excitation for a Raman instrument presents several technical difficulties. The off-the-shelf components for a UV laser-based instrument are usually inferior in nominal characteristics when compared with the same components available for VIS/NIR. Overall technical development of UV-suitable components lags behind the developments of components for the NIR spectral region. One of the most important shortcomings in a UV-based Raman system is the poor performance in recording spectral information close to the Rayleigh line, namely the Raman bands up to $500 \mathrm{~cm}^{-1}$ from the excitation laser line. This spectral window is of utmost importance since it covers a significant part of the "fingerprint region" in the Raman spectra. For most of the inorganic materials the identification of a component is heavily based on this fingerprint spectral area which usually spans up to $1,200 \mathrm{~cm}^{-1}$ (relative wave numbers).

\subsection{Raman Optical Head and Rayleigh Filters}

Figure 5 shows the schematic optical path of a Raman optical head. The laser excitation is transmitted via a lens pair to the sample surface. A clever design of band pass in the excitation path and long pass filters in the reception path allow for minimizing the Raman signal generated within the fibre itself. Unfiltered, these quartz lines overlap with the Raman spectrum of the sample and thus reduce the $\mathrm{S} / \mathrm{N}$ drastically.

Additionally, plasma lines of the laser will not reach the sample, which would generate second-order spectra. The dichroic mirror or notch filter separates the Raman signal from parasitic or disturbing Raleigh signals and laser plasma lines. The important parameters to be considered in the design of the Raman optical head are:

- High filter efficiency

- High thermal stability and insensitivity to space/Mars environment

- High signal-to-background ratio 


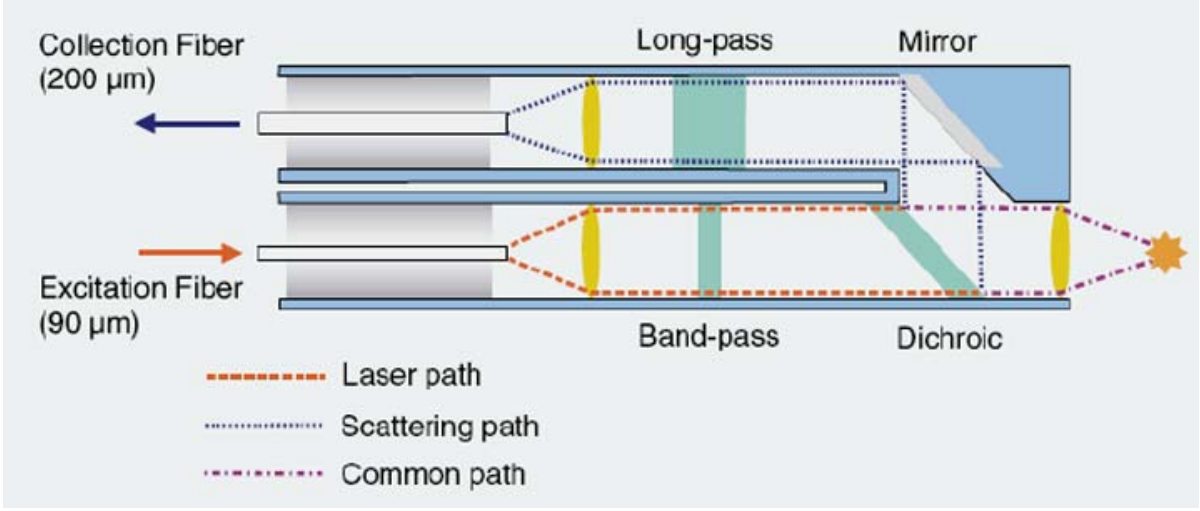

Fig. 5 Fibre-coupled Raman head, typical commercial fibre probe configuration by InPhotonics

- Maximal transmission for the spectral window of the expected Raman signal

- High numerical aperture to lower the requirements for laser power and spectrometer sensitivity

- Minimal diameter of the lenses used in the probe (which will dictate the overall size and mass of the probe).

For NIR Raman @ 785 nm a number of commercial optical heads are available; e.g., the MR Probe from Kaiser Optical Systems.

Since the Raman instrument needs an imaging lens to focus the spatial resolution, a combination of the Raman instrument with a microscope system (used for white light imaging) can be advantageous.

Similar to the technical challenges facing laser and spectrometer design, the optical design for the beam splitting and notch/edge filters inside the Raman head becomes more challenging with deeper wavelengths. In the VIS and the NIR, a number of commercial, steep-edge notch filters and beam splitters are available. The holographic notch filters at longer wavelengths in particular show supreme performance. A selection with regard to temperature stability and space environment is required. The availability of commercial filters is drastically reduced within the UV wavelength range, especially in the deep UV range; currently, there are no commercial notch filters to meet this need on the market. As compact deep UV lasers are still rare on the market, suitable filters still have to be developed.

An interesting new approach to filtering the laser back reflection between the Raman head and the spectrometer is to introduce a fibre Bragg grating in the reception fibre. Typically these resonant gratings allow extremely sharp reflection peaks if they are used with single-mode fibres. A Bragg grating applied to a multimode fibre is capable of blocking a wavelength band of approx. $1 \mathrm{~nm}$ with about $25 \mathrm{~dB}$. This greatly assists the filtering in the optical head (independent from the selected Raman wavelength) and improves the $\mathrm{S} / \mathrm{N}$ of the signal significantly.

\subsection{Spectrometer and Detector (Spectral Sensor)}

Different types of spectral sensors for Raman spectroscopy have been developed with the aim of meeting the tight requirements imposed by a space mission. Dispersive spectrometers with multichannel detectors (CCDs) (Wang et al. 1999; Dickensheets et al. 2000; 
Fig. 6 Miniaturized Hadamard-Transform-Spectrometer

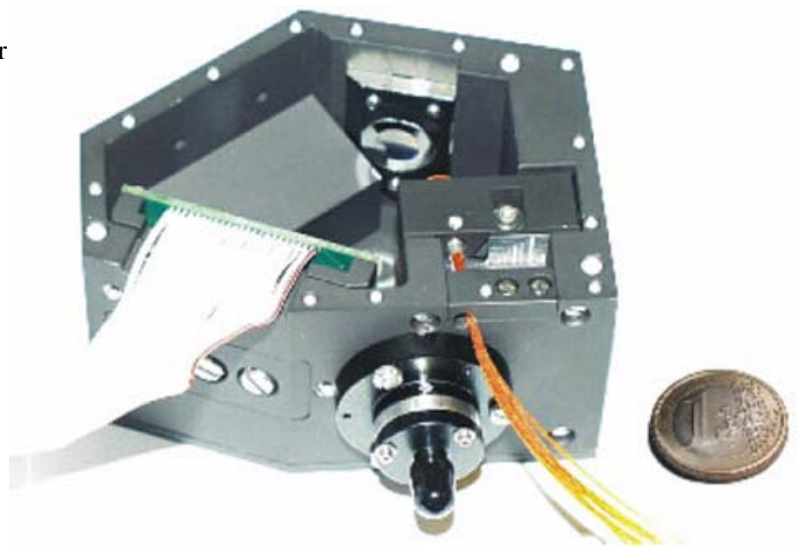

Maurice et al. 2004; AURORA/EXOMARS 2005; Mugnuolo et al. 2000) and also tuneable monochromators with single-channel detectors (APD, PMT) (Tarcea et al. 2002; Popp et al. 2003) have been used as spectral sensors. The vast majority of the developed systems so far are based on dispersive spectrometers, which offer the advantage of multichannel detection when combined with a CCD detector. Scientific detectors with sufficient sensitivity for Raman detection typically have pixel pitches of $13 \mu \mathrm{m}$ or more. For typical dispersive spectrometers and Echelle spectrometers, where the necessary minimum linear dispersion in the best case is determined by the required spectral resolution and the pixel pitch of the detector, this leads to relatively large linear dispersions and consequently to limitations in compactness.

A novel and promising approach is the Hadamard-Transform-Spectrometer. Its high efficiency is achieved by combining a specially adapted grating with a multislot array, operating as the subsystem aperture. The combination of the multislot array, the adapted grating and the CCD generates so-called "sub-pixels" which enhance the spectral resolution considerably.

To generate subpixel information, the spectrum is measured multiple times with slightly different sampling rasters, shifted by fractions of the physical pixel pitch. The latter is achieved by changing the position of the entry slit with respect to the spectrometer, leading to an equivalent shift of the spectral image on the detector. This can be done by physically shifting the slit and then taking successive measurements (time multiplex) or by arranging slightly shifted slits perpendicular to the direction of dispersion.

The Hadamard-Transform-Spectrometer architecture provides a significantly higher throughput along with improved sensitivity and spectral resolution compared to commercial spectral sensors. The detector consists of a CCD matrix with a length of $25.6 \mathrm{~mm}$. The resolution of $5 \mathrm{~cm}^{-1}$ is reached for the whole spectral range with a total length for the Hadamard spectrometer component of about $55 \mathrm{~mm}$ (Fig. 6) (Riesenberg et al. 2002). For the detection of very weak signals a cooling of the CCD is necessary. These types of spectral sensors were developed by a team led by Prof. Riesenberg at Institute of Photonic Technology in Jena.

Systems where the spectrometer core is a tuneable monochromator-e.g., an acoustooptic tuneable filter (AOTF) or a liquid-crystal tuneable filter (LCTF), have also been investigated (Tarcea et al. 2002; Popp et al. 2001). An important characteristic of these systems is the ability to transmit images and therefore the use as imaging spectrometers is possible. 


\section{References}

AURORA/EXOMARS, Combined Raman/LIBS Spectrometer Elegant Bread Board Technical Proposal Document. TNO Science and Industry, 2005

S. Chadha, R. Manoharan, P. Moënne-Loccoz, W.H. Nelson, W.L. Peticolas, J.F. Sperry, Appl. Spectrosc. 47, 38-43 (1993)

W.D. Cochran, Adv. Space Res. 1, 143 (1981)

D.L. Dickensheets, D.D. Wynn-Williams, H.G.M. Edwards, C. Schoen, C. Crowder, E.M. Newton, J. Raman Spectrosc. 31(7), 633-635 (2000)

H.G.M. Edwards, D.W. Farwell, M.M. Grady, D.D. Wynn-Williams, P. Wright, 1, Planet. Space Sci. 47, 353-363 (1999)

A. Ellery, D. Wynn-Williams, Astrobiology 3, 565 (2003)

A. Ellery, D. Wynn-Williams, J. Parnell, H.G.M. Edwards, D. Dickensheets, J. Raman Spectrosc. 35, 441-457 (2004)

P.A. Estec, J.J. Kovach, P. Waldstein, C. Karr Jr., Proc. Lunar Sci. Conf. 3, 3047-3067 (1972)

L.A. Haskin, A. Wang, K.M. Rockow, B.L. Jolliff, R.L. Korotev, K.M. Viskupic, J. Geophys. Res. [Planets] 102(E8), 19293-19306 (1997)

E.J. Isreal, R.E. Arvidson, A. Wang, J.D. Pasteris, B.L. Jolliff, J. Geophys. Res. [Planets] 102(E12), 2870528716 (1997)

V. Klein, J. Popp, N. Tarcea, M. Schmitt, W. Kiefer, S. Hofer, T. Stuffler, M. Hilchenbach, D. Doyle, M. Dieckmann, J. Raman Spectrosc. 35, 433-440 (2004)

R.L. Korotev, A. Wang, L.A. Haskin, B.L. Jolliff, Lunar and Planetary Science XXIX, 29. Lunar and Planetary Science Conference, 1998, pp. 1797-1798

P.G. Lucey et al., LPSC XXIX (1998), Abstract \#1354

S. Maurice, F. Rull, EXLIBRIS (EXOMARS Laser Induced Breakdown Spectroscopy-Raman Integrated Spectrometers), EGU 1 General Assembly, Abstract EGU04-A-06349, April 2004. See details at http://www.esa.int/specials/aurora/

P.F. McMillan, J. Dubessy, R. Hemley, in Raman Microscopy, ed. by G. Turrell, J. Corset (Academic, London, 1996), p. 289

A.K. Misra et al., Spectrochimica Acta A 61, 2281-2287 (2005)

R. Mugnuolo, F. Angrilli, S. Debei, E. de Marchi, A. Nita, A. Terribili, Presented at the 6th ESA Workshop on Advanced Space Technology for Robotics and Automation (ASTRA 2000), ESA-ESTEC, Noordwijk, The Netherlands, November 2000

J. Popp, W. Kiefer, Encyclopedia of Analytical Chemistry (Wiley, 2000), pp. 13104-13142

J. Popp, N. Tarcea, W. Kiefer, M. Hilchenbach, N. Thomas, S. Hofer, T. Stuffler, ESA Publication. ESA SP-496, 2001, pp. 193-196

J. Popp, N. Tarcea, M. Schmitt, W. Kiefer, R. Hochleitner, G. Simon, M. Hilchenbach, S. Hofer, T. Stuffler, in Proceedings of the Second European Workshop on Exo/Astrobiology, 2002, p. 339

J. Popp, N. Tarcea, M. Schmitt, W. Kiefer, M. Hilchenbach, J. Whitby, N. Thomas, T. Stuffler, S. Hofer, H. Edwards, F. Rull, Presented at the 37th ESALAB Symposium, ESA-ESTEC, Noordwijk, The Netherlands, 1-3 July 2003

L. Richter, P. Costeb, V.V. Gromovc, H. Kochana, R. Nadalinid, T.C. Nge, S. Pinnaf, H.E. Richtera, K.L. Yungg, Planet. Space Sci. 50(9), 903-913 (2002)

R. Riesenberg, G. Nitzsche, A. Wuttig, B. Harnisch, Smaller Satellites: Bigger Business (Kluwer, 2002), pp. 403-406

F. Rull, J. Martinez-Frias, Spectrosc. Eur. 18(1), 21 (2006)

S.K. Sharma, S.M. Angel, M. Ghosh, H.W. Hubble, P.G. Lucey, Appl. Spectrosc. 56, 699 (2002)

S.K. Sharma, P.G. Lucey, M. Ghosh, H.W. Hubble, K.A. Horton, Spectrochimica Acta A 59, 2391-2407 (2003)

S.K. Sharma et al., Spectrochimica Acta A 59, 2391-2407 (2003)

Stoper et al., Proc. SPIE 5163, 99-110 (2004)

M.C. Storrie-Lombardi, A.I. Tsapin, G.D. McDonald, H. Sun, K.H. Nealson, in Book of Abstracts, 217th ACS National Meeting, Anaheim, CA, March 21-25, vol. GEOC-069 (American Chemical Society, Washington, 1999)

M.C. Storrie-Lombardi, W.F. Hug, G.D. McDonald, A.I. Tsapin, K.H. Nealson, Rev. Sci. Instrum. 72(12), 4452-4459 (2001)

N. Tarcea, J. Popp, M. Schmitt, W. Kiefer, R. Hochleitner, G. Simon, M. Hilchenbach, S. Hofer, T. Stuffler, in Proceedings of the Second European Workshop on Exo/Astrobiology, 2002, p. 399 
N. Tarcea, J. Popp, M. Schmitt, W. Kiefer, T. Stuffler, S. Hofer, G. Simon, R. Hochleitner, B. Hofmann, M. Hilchenbach, EGS - AGU - EUG Joint Assembly, Nice, France, April 2003, p. 11790

A. Wang, L.A. Haskin, E. Cortez, Appl. Spectrosc. 52, 477 (1998)

A. Wang, B.L. Joliiiff, L.A. Haskin, J. Geophys. Res. [Planets] 104(E4), 8509-8519 (1999)

A. Wang, L.A. Haskin, A.L. Lane, T.J. Wdowiak, S.W. Squyres, R.J. Wilson, L.E. Hovland, K.S. Manatt, N. Raouf, C.D. Smith, J. Geophys. Res. 108, 5(1) (2003) 